\title{
The scattering of 3D sound sources by rigid barriers in the vicinity of tall buildings
}

\author{
L. Godinho*, J. António, A. Tadeu \\ Department of Civil Engineering, University of Coimbra, Polo II-Pinhal de Marrocos, 3030-290 Coimbra, Portugal
}

Received 20 December 2001; accepted 6 March 2002

\begin{abstract}
The acoustic scattering of a three-dimensional (3D) sound source by an infinitely long rigid barrier in the vicinity of a tall building is analyzed using the boundary element method (BEM). The acoustic barrier is modeled using boundary elements, and is assumed to be nonabsorbing, while the image source method is used to model the tall building as an infinite vertical barrier. A frequency domain BEM formulation is used, and time domain responses are then obtained by applying an inverse Fourier transformation.

Since the geometry of the problem does not vary along one direction, the 3D solution can be calculated as the summation of a sequence of 2D problems, each solved for a different spatial wavenumber, $k_{z}$. To obtain the $3 \mathrm{D}$ solution, a discrete form wavenumber transform is performed by considering an infinite number of virtual point sources equally spaced along the $z$ axis. Complex frequencies are used to minimize the influence of these neighboring fictitious sources.

Numerical simulations are performed using barriers of varying sizes, evaluating the attenuation of the sound pressure level in the vicinity of the building façade. The creation of shadow zones by the barriers is analyzed and time responses are presented to better understand the sound propagation around these obstacles. (C) 2002 Elsevier Science Ltd. All rights reserved.
\end{abstract}

Keywords: Acoustic barrier; Sound propagation; Tall buildings

\section{Introduction}

Diffraction-based methods have been used by many authors as a tool to analyze sound propagation in the presence of obstacles. Lam [1] introduced one such method for the calculation of the acoustic energy loss produced by the insertion of simple, finite length three-dimensional (3D) acoustic barriers. This work was later extended by Muradali and Fyfe [2] to include the modeling of 2D geometries.

Precision can be improved by using numerical methods like the boundary element method (BEM) or the finite element method to solve the wave-equation for each frequency. Based on the theory of slender bodies, Filippi and Dumery [3] and Terai [4] developed a boundary integral equation technique to analyze the scattering of sound waves by thin rigid screens in unbounded regions. This method was subsequently extended by Kawai and Terai [5] to allow the prediction of sound attenuation by rigid barriers over a totally reflective ground surface. A 2D boundary element technique was used by Morgan et al. [6] to assess the influence of the shape and absorbent surface of railway

\footnotetext{
* Corresponding author.
}

noise barriers. Their work compared a boundary element prediction for simple barrier and vehicle shapes with results given by the standard UK prediction method. Lacerda et al. [7] proposed a dual boundary element formulation for analyzing the $2 \mathrm{D}$ sound propagation around acoustic barriers, over an infinite plane, in which both the ground and the barrier were considered to be absorptive. The 3D propagation of sound around an absorptive barrier was studied by Lacerda et al. [8], introducing a dual boundary element formulation that allowed the barrier to be modeled as a simple surface.

The present work takes into account both the influence of the acoustic barrier and the presence of very large buildings next to it. The pressure field generated by wave scattering at both objects is calculated using a standard boundary element formulation. Both the acoustic barrier and the building are considered to be totally reflective.

In our model, the acoustic barrier is considered to be of infinite length, while the acoustic source takes the form of a point load. This situation is usually referred to as a two-anda-half-dimensional problem, for which solutions can be obtained by means of a spatial Fourier transform in the direction in which the geometry does not vary [9]. 
The BEM model is used to compute the 3D pressure field generated by a point pressure source in the vicinity of a rigid barrier placed between the source and a tall building. The building is treated as an infinite rigid vertical plane surface. Simulation analyses are performed to investigate wave propagation in the vicinity of such buildings in the presence of neighboring varying-sized acoustic barriers. Both frequency and time domain responses are obtained to permit a quantitative study of the $3 \mathrm{D}$ effects of the scattering.

\section{Problem definition}

The 3D pressure field generated by a harmonic point load inside a uniform acoustic medium is given by

$$
p_{\text {inc }}=\frac{A \exp \left(\mathrm{i} \frac{\omega}{\alpha}\left(\alpha t-\sqrt{\left(x-x_{0}\right)^{2}+\left(y-y_{0}\right)^{2}+z^{2}}\right)\right)}{\sqrt{\left(x-x_{0}\right)^{2}+\left(y-y_{0}\right)^{2}+z^{2}}}
$$

where $\omega$ is the excitation frequency, $\left(x_{0}, y_{0}, 0\right)$ the position of the load, the subscript inc denotes the incident field, $A$ the wave amplitude, $\alpha$ the pressure wave velocity of the medium, and $\mathrm{i}=\sqrt{-1}$.

Applying a Fourier transformation in the $z$ direction to Eq. (1), and defining the effective wavenumbers by $k_{\alpha}=$ $\sqrt{\left(\omega^{2} / \alpha^{2}\right)-k_{z}^{2}}$ with $\operatorname{Im} k_{\alpha}<0$, where $k_{z}$ is the axial wavenumber, one obtains

$\hat{p}_{\text {inc }}\left(\omega, x, y, k_{z}\right)=\frac{-\mathrm{i} A}{2} H_{0}^{(2)}\left(k_{\alpha} \sqrt{\left(x-x_{0}\right)^{2}+\left(y-y_{0}\right)^{2}}\right)$

where $H_{n}^{(2)}(\cdot)$ are second Hankel functions of order $n$.

Assuming the existence of an infinite set of sources, evenly spaced along the $z$ direction, the $3 \mathrm{D}$ incident field can be written as

$p_{\text {inc }}(\omega, x, y, z)=\frac{2 \pi}{L} \sum_{m=-\infty}^{\infty} \hat{p}_{\text {inc }}\left(\omega, x, y, k_{z}\right) \mathrm{e}^{-\mathrm{i} k_{z} z}$

where $L$ is the spatial source interval, and $k_{z}=(2 \pi / L) m$. The $3 \mathrm{D}$ pressure field may now be calculated as the pressure irradiated by a sum of harmonic (steady-state) line loads with amplitudes varying sinusoidally in the 3D. This sum converges and can be approximated by a finite number of terms.

In the present paper, a spatially uniform acoustic medium bounded by two perpendicular flat surfaces is modeled. One of these surfaces simulates the horizontal rigid flat floor, while the other represents the façade of the tall building. Inside the acoustic medium, the presence of a vertical rectangular rigid obstacle (acoustic barrier) is assumed. When the vertical plane and the horizontal plane are defined by $x=0$ and $y=0$, respectively, the acoustic pressure field (Green's function $G\left(x, x_{0}, \omega\right)$ ) can be computed by the following expression:

$G\left(x, x_{0}, \omega\right)=\sum_{j=1}^{\mathrm{NS}} \frac{-\mathrm{i}}{4}\left[H_{0}\left(k_{\alpha} r_{j}\right)\right]$

with $\mathrm{NS}=4$, and

$r_{1}=\sqrt{\left(x-x_{0}\right)^{2}+\left(y-y_{0}\right)^{2}}$

$r_{2}=\sqrt{\left(x-x_{0}\right)^{2}+\left(y+y_{0}\right)^{2}}$

$r_{3}=\sqrt{\left(x+x_{0}\right)^{2}+\left(y-y_{0}\right)^{2}}$

$r_{4}=\sqrt{\left(x+x_{0}\right)^{2}+\left(y+y_{0}\right)^{2}}$

\section{Boundary element formulation}

The acoustic BEM formulation used in this work is well known, and so the details of its formulation are omitted. However, it is important to state that the solution of each 2D problem requires the evaluation of the integral

$H^{k l}=\int_{C_{l}} H\left(\underline{x}_{k}, \underline{x}_{l}, n_{l}\right) \mathrm{d} C_{l}$

where $H^{k l}$ is the pressure velocity component at $\underline{x}_{k}$ due to pressure load at $\underline{x}_{l}$ and $n_{l}$ is the unit outward normal for the $l$ th boundary segment $C_{l}$. The Green's function for pressure velocity can be obtained by differentiating Eq. (4) in relation to the unit outward normal.

Gauss-Legendre quadrature is used to perform the required integrations in Eq. (5), using no fewer than six integration points. In this work, the acoustic barrier is modeled as a thick object, and, to maintain accuracy, the required numerical integrations on elements close to or directly facing the loaded element are performed using higher order Gauss-Legendre integration schemes.

The pressure field inside the acoustic medium can then be calculated in relation to the nodal pressure values obtained.

\section{Pressure in time-space}

After calculating the frequency domain responses, time signals are obtained by means of an inverse Fourier transformation in $\omega$. The acoustic source used is assumed to have a temporal variation given by a Ricker pulse:

$u(\tau)=A\left(1-2 \tau^{2}\right) \mathrm{e}^{-\tau^{2}}$

where $A$ is the amplitude, $\tau=\left(t-t_{\mathrm{s}}\right) / t_{0}$ and $t$ represents the time, $t_{\mathrm{s}}$ the time when the maximum occurs, while $\pi t_{0}$ the dominant wavelet period. By applying a Fourier 
transformation to this function, one obtains:

$U(\omega)=A\left[2 \sqrt{\pi t_{0}} \mathrm{e}^{-\mathrm{i} \omega t_{\mathrm{s}}}\right] \Omega^{2} \mathrm{e}^{-\Omega^{2}}$

where $\Omega=\omega t_{0} / 2$.

The inverse Fourier transformations required for the calculation of both the time and the 3D frequency domain responses are performed by means of a summation of a finite number of terms, either in frequency or spatial wavenumbers. This is mathematically equivalent to assuming the existence of periodic sources placed at spatial intervals of $L=2 \pi / \Delta k_{z}$ along $z$, and temporal intervals of $T=2 \pi / \Delta \omega$. In these expressions, $\Delta k_{z}$ and $\Delta \omega$ represent the wavenumber and frequency increment, respectively.

To prevent the periodic sources from contaminating the response, the spacing between them must be sufficient to ensure that their contribution arrives at times later than $T$. To help achieve this goal, the frequency axis is shifted slightly downwards in the complex plane, using complex frequencies with an imaginary part of the form $\omega_{\mathrm{c}}=\omega-$ in (with $\eta=0.7 \Delta \omega$ ).

\section{Validation of the BEM algorithm}

The BEM algorithm used was applied to calculate the response around a cylindrical circular rigid pipe placed inside an unbounded homogeneous acoustic medium $(\alpha=$ $340 \mathrm{~m} / \mathrm{s}$ ) and illuminated by a harmonic point pressure load. To obtain the required Green's function, the NS parameter in Eq. (4) must be set to one. For this geometric configuration, closed form solutions are well known, making it possible to validate the numerical algorithm.

The results (not presented) showed an extremely accurate BEM response for low frequencies and revealed only slight differences at higher frequencies.

\section{Numerical examples}

The influence of the presence of an acoustic barrier placed between a point pressure load and a very tall building has been assessed using the method described. In all the examples presented, the ground surface and the building are modeled as non-absorbing surfaces, and a pressure wave velocity of $340 \mathrm{~m} / \mathrm{s}$ was ascribed to the host acoustic medium. An acoustic point source is placed $25.0 \mathrm{~m}$ away from a tall building and $0.6 \mathrm{~m}$ above the ground. An acoustic barrier of height $h$ is inserted between the source and the façade, with its axis $5.0 \mathrm{~m}$ away from the source, to reduce the acoustic sound registered on the façade. The geometry of this problem is represented in Fig. 1.

The acoustic barrier is modeled as a $0.2 \mathrm{~m}$ thick body. It is discretized with an appropriate number of boundary elements, defined by setting the relation between the wavelength and the length of each boundary element to 8 .

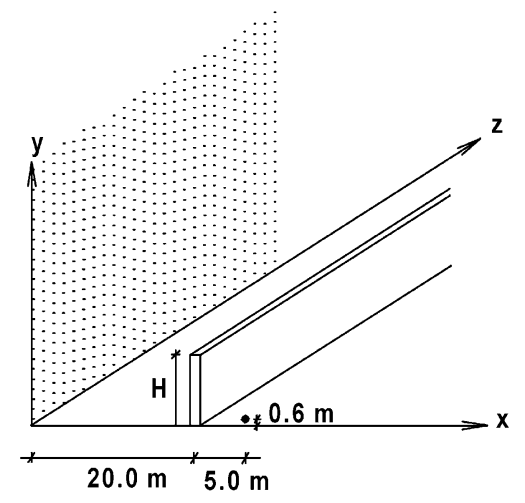

Fig. 1. Geometry of the problem.

However, in no case is the number of boundary elements used less than 32. A first series of simulations was performed to calculate the response over a grid of receivers placed along a vertical plane, parallel to the building façade and $0.5 \mathrm{~m}$ away from it, equally spaced at a distance of 1.0 and $4.0 \mathrm{~m}$ apart along the vertical and longitudinal directions, respectively. Responses were calculated for frequencies in the range $2-256 \mathrm{~Hz}$, with a frequency increment of $2 \mathrm{~Hz}$. The frequency increment used determined a total time window response of $0.5 \mathrm{~s}$. The source is assumed to emit a Ricker pulse with a characteristic frequency of $100 \mathrm{~Hz}$.

The sound pressure level $\left(10 \log \left[p^{2} /\left(2 \times 10^{-5}\right)^{2}\right]\right.$, where $p$ is the maximum amplitude of the time responses calculated for each receiver) obtained when there is no barrier is represented in Fig. 2. A gray scale is used to represent the pressure level, ascribing lighter and darker shades to higher and lower values of the pressure level, respectively. The results show that the maximum sound pressure level field does not occur at $z=0.0 \mathrm{~m}$. This can be explained by the effect of the directly incident pulses being added to those reflected on the building. This behavior does not occur if the grid of receivers is positioned at $x=0.0 \mathrm{~m}$ (not illustrated). A decrease in the general sound pressure level is noted as the distance of the receiver to the source increases.

Fig. 3(a) and (b) gives the sound pressure level, and the attenuation produced by the insertion of an acoustic barrier between the source and the building. When the barrier is $2.0 \mathrm{~m}$ tall, these results indicate a poorer performance of the barrier for receivers placed closer to the ground, due to the interaction between the direct field diffracted by the barrier and that reflected by the rigid ground floor. Receivers placed at greater distances from the floor register an improvement in the performance of the barrier, with maximum efficiency being reached at approximately $8 \mathrm{~m}$ above the ground for $z=0.0 \mathrm{~m}$. Results obtained at receivers placed further from the ground show a progressive loss of efficiency, and, at greater heights from the ground, the presence of the barrier can even lead to an amplification of the response. The acoustic barrier does not have a constant performance along the $z$ axis. The points of maximum efficiency for 

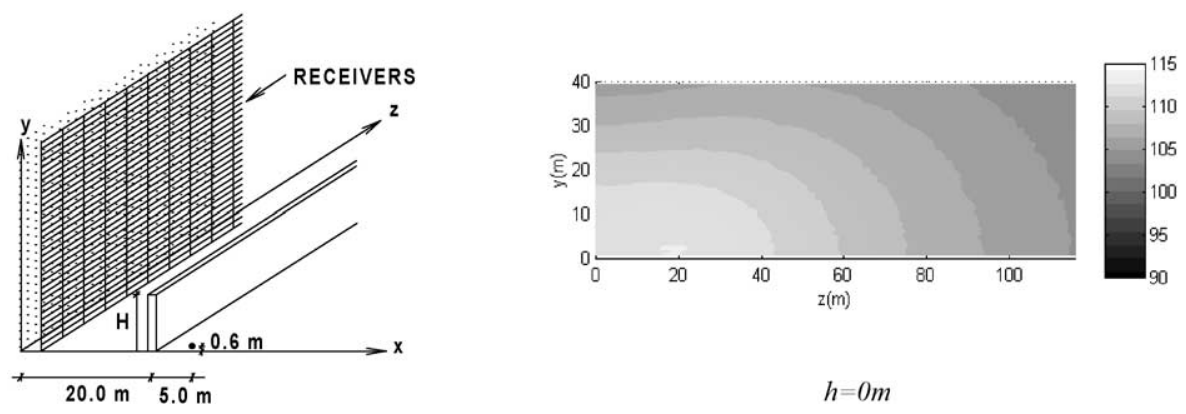

Fig. 2. Sound pressure level along a vertical plane $0.5 \mathrm{~m}$ from the building.

consecutive vertical $z$ planes form an inclined line, which indicates better performances at receivers placed further above the floor as $z$ increases. This behavior indicates that the reflections on the rigid ground close to the building, mentioned earlier, increase in importance as $z$ increases.

Increasing the height of the barrier from 2.0 to $4.0 \mathrm{~m}$ and then to $6.0 \mathrm{~m}$, there is a global increase in the sound pressure level attenuation for the full domain of receivers. The line of maximum efficiency described earlier is now closer to the ground surface, and less inclined. It appears that, as the height of the barrier increases, the sound waves reflected on the rigid ground close to the building and the direct field diffracted by the barrier, both lose importance relative to the receivers closer to the floor.

Next, to better illustrate the propagation of the sound pressure from its source to the receivers, the time responses are presented for receivers placed at $z=0.0$ and $30.0 \mathrm{~m}$.

Fig. 4(a) displays the time responses at receivers placed at $z=0.0 \mathrm{~m}$ (source plane), for barriers 0.0, 2.0, 4.0, and $6.0 \mathrm{~m}$ in height. These records in the time domain exhibit a series of incident pulses and the result of their reflections on
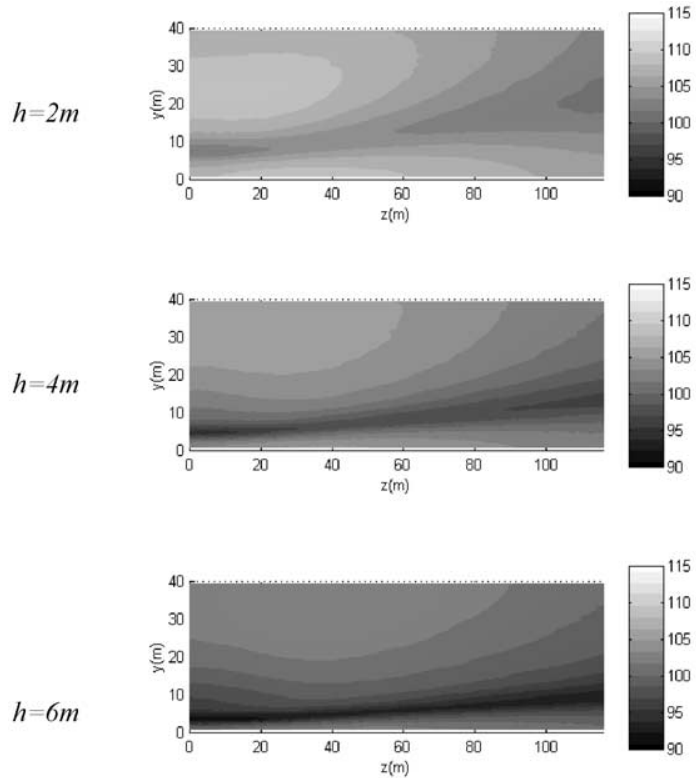

a) the ground, wall, and barrier. The results show that the first set of pulses is recorded at later times at receivers closer to the ground, as taller barriers are used. A pulse therefore takes longer to travel from the source to the edge of the barrier and then to these receivers as the height of the obstacle increases. The arrival times of the different pulses agree with those calculated using the acoustic ray theory, allowing identification of the travel paths followed by the different pulses shown in the response. It can further be seen that the amplitude of the response recorded at these receivers becomes smaller as a result of a diffraction effect at the edge of the barrier. As described earlier, the efficiency of the acoustic barrier is seen to increase as its height increases.

A second pulse is clearly visible in the time responses, just after the first arrival, when an acoustic barrier is present. This second pulse is clearly separated from the first when the barrier is taller and the receivers are placed at greater distances from the ground. This second pulse is caused by a prior reflection from the rigid ground.

As time progresses, a third pulse appears when there is an
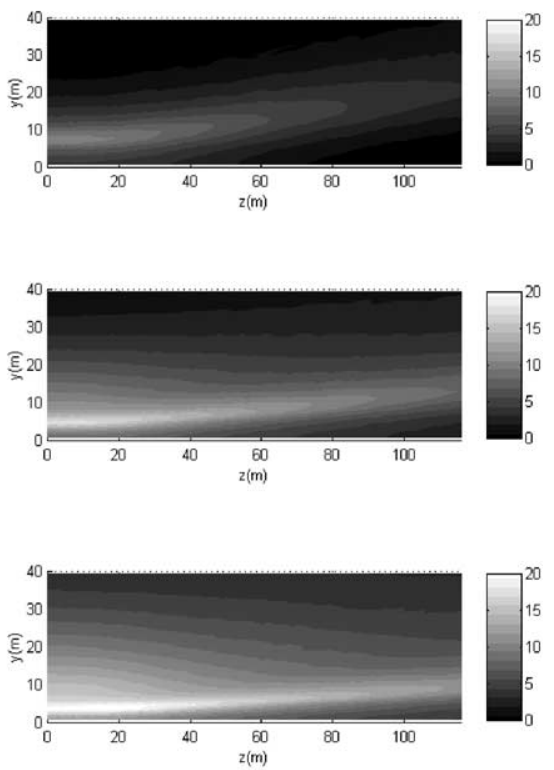

b)

Fig. 3. Sound pressure along a vertical plane $0.5 \mathrm{~m}$ from the building: (a) pressure level; (b) pressure attenuation. 

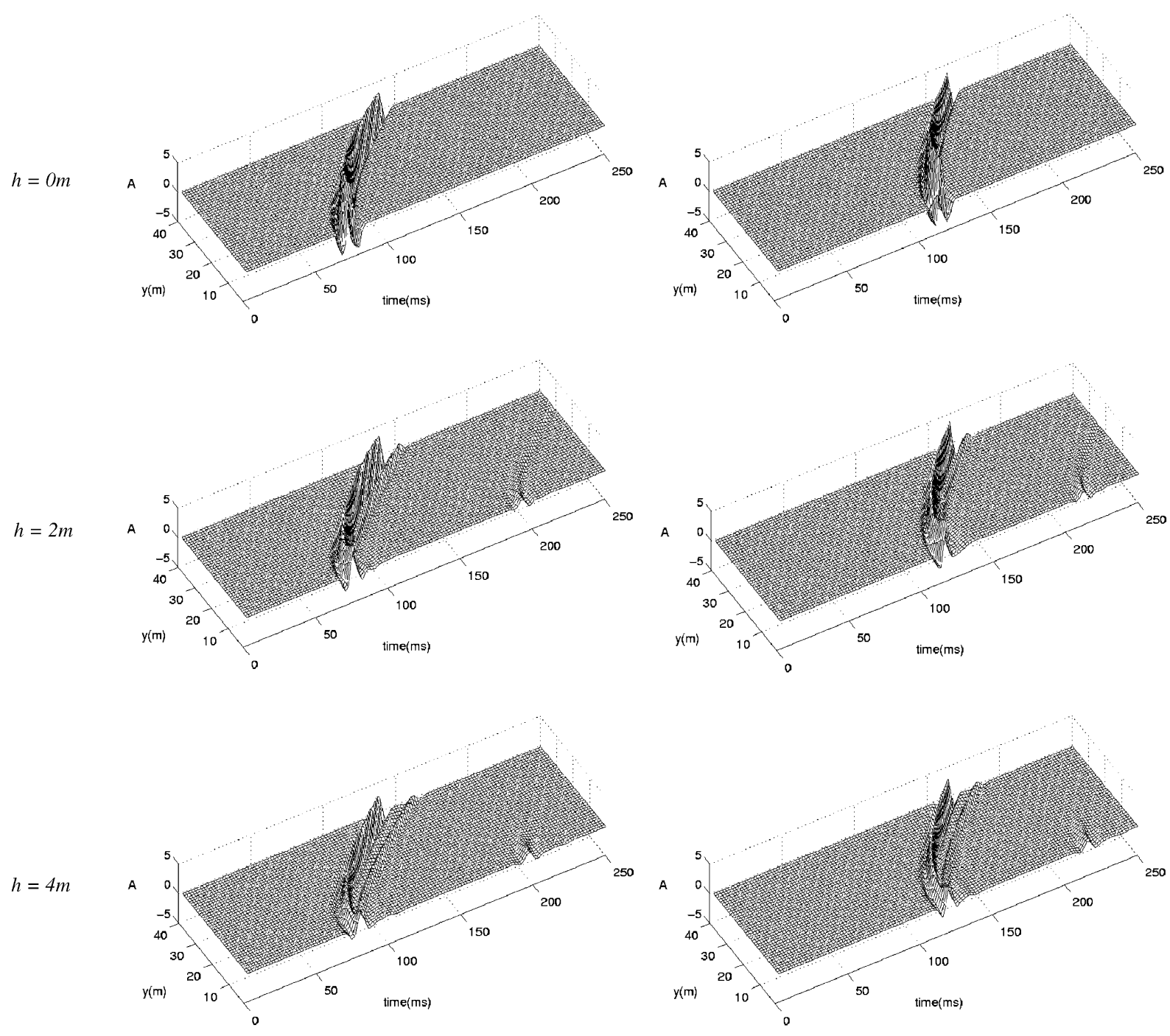

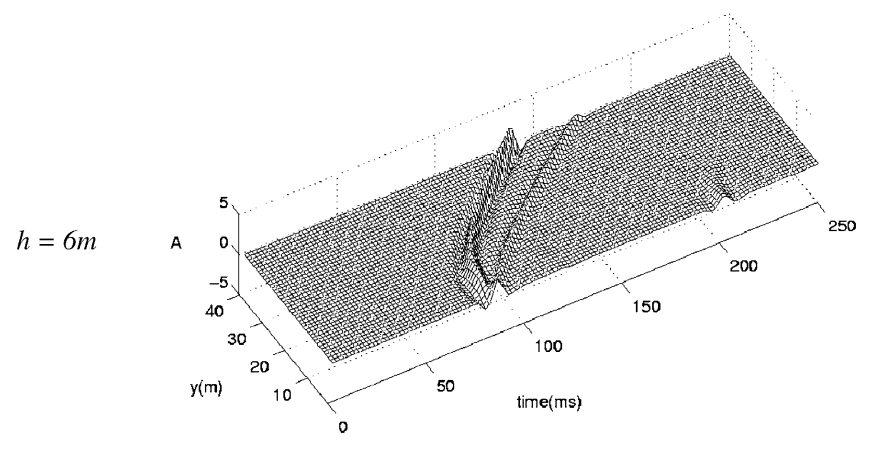

(a)

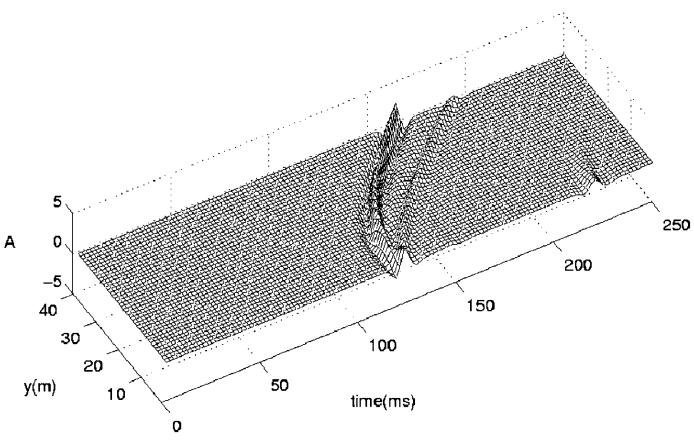

(b)

Fig. 4. Time response at receivers $0.5 \mathrm{~m}$ from the building: (a) $z=0.0 \mathrm{~m}$; (b) $z=30.0 \mathrm{~m}$.

acoustic barrier inserted between the source and the building. This pulse originates in the energy trapped between the barrier and the building, which allows secondary reverberation effects to occur. Other pulses occur at later times (not visible in the time window presented) as a result of this interaction between the barrier and the rigid wall. The approximate time difference between these pulses, showing progressively lower amplitude as energy dissipates, is given by $40.0 \mathrm{~m} / 340.0(\mathrm{~m} / \mathrm{s})=$ $117.6 \mathrm{~ms}$.

Fig. 4(b) illustrates the responses obtained for the same set of receivers for $z=30.0 \mathrm{~m}$. The different pulses arrive at later times because they travel along longer paths. However, the results exhibit features similar to the ones observed for 

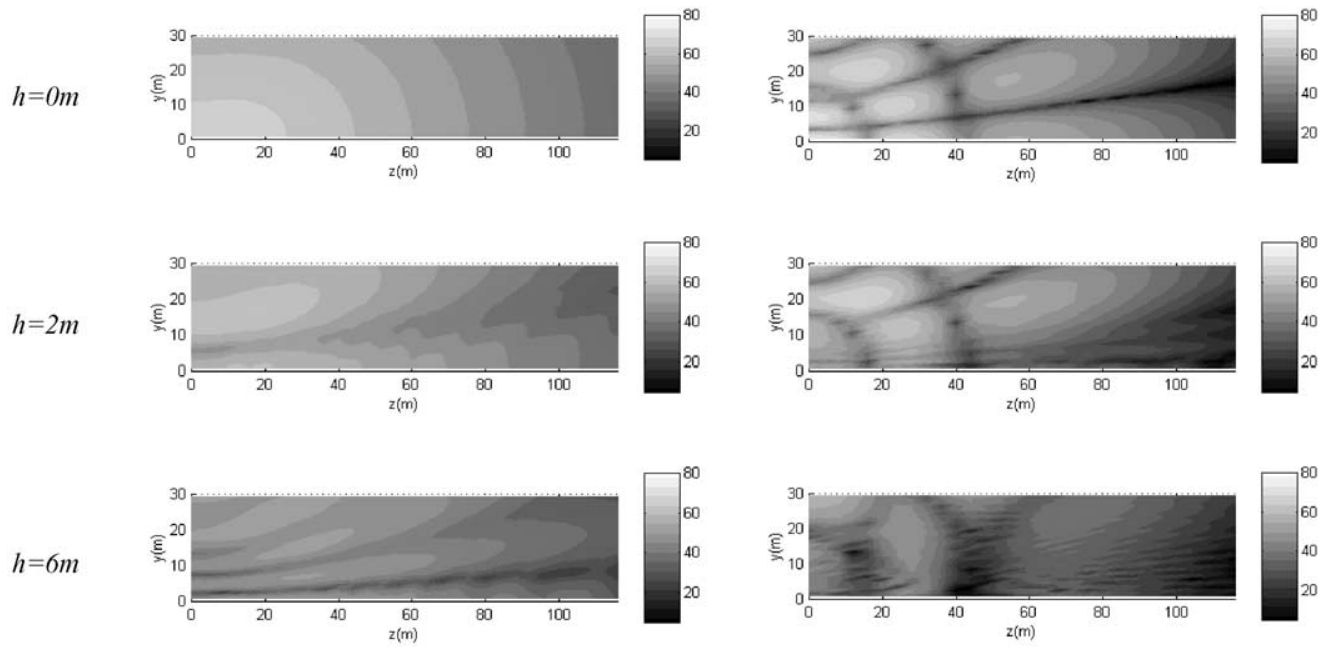

a)

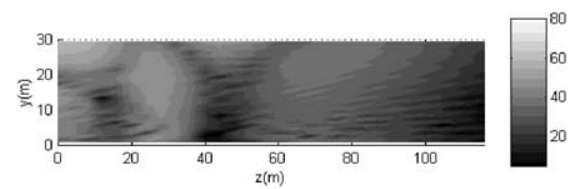

b)

Fig. 5. Sound pressure along a vertical plane $0.5 \mathrm{~m}$ from the building: (a) frequency of $125 \mathrm{~Hz}$; (b) frequency of $1000 \mathrm{~Hz}$.

$z=0.0 \mathrm{~m}$. Comparison of the responses for $z=0.0$ and $30.0 \mathrm{~m}$ reveals a very slight drop in amplitude. This can be explained by the effect of the directly incident pulses being added to those reflected on the building, already mentioned earlier.

Analyses in the frequency domain were performed for the same geometries. Sound pressure levels are shown in Fig. 5, for the frequencies of 125 and $1000 \mathrm{~Hz}$. In the absence of the acoustic barrier, the pressure field results from the direct incident field interacting with that reflected by the floor and the building. The total field is thus given by the sum of waves with different phases leading to a spatially variable sound pressure level, distinguishable as a pattern of darker and lighter zones. This phenomenon becomes more complex as the frequency increases.

Again, the performance of the $2.0 \mathrm{~m}$ barrier is poorer at receivers placed closer to the floor when the excitation frequency is low. The reflected field on the ground and the trapped energy between the barrier and the building are responsible for this behavior. As the frequency increases, the barrier creates a 'shadow' zone behind it, leading to a pronounced attenuation of the sound pressure field at the lower receivers. The influence of the reflections at receivers very close to the ground is, however, maintained.

The shadow created behind the barrier becomes more intense as the height of the barrier changes from 2.0 to $6.0 \mathrm{~m}$, and as the frequency increases from 125.0 to $1000.0 \mathrm{~Hz}$. This behavior is expected since higher frequency waves have smaller wavelengths, and are easily influenced by smaller obstacles.

\section{Conclusions}

The analysis of the sound pressure level obtained over a plane parallel to the building indicates that there is no uniform performance on the part of the acoustic barrier. The barrier achieved maximum efficiency at receivers placed at greater distances from the floor as $z$ increased. The taller the barrier the greater the attenuation of the sound pressure level, for the full domain of receivers, with a maximum efficiency being found for receivers nearer the ground.

It was shown that the time arrivals of the different pulses at the receivers agree with the travel path of the incident pulses and their reflections on the ground, wall and barrier. The results confirm that the efficiency of the acoustic barrier was poorer the nearer the receiver was to the ground, owing to the effect of the interaction of the different pulses: diffracted by the barrier and reflected on the ground.

Analysis of frequency domain responses shows that, for a low excitation frequency, the performance of the acoustic barrier was poorer at receivers placed in the close vicinity of the floor. As the excitation frequency increased, the barrier created a shadow zone behind it, leading to a marked drop of the sound pressure field recorded at lower receivers. The attenuation provided by the barrier could be further improved by increasing its height to create a more intense shadow zone behind it.

\section{References}

[1] Lam YW. Using Maekawa's chart to calculate finite length barrier insertion loss. Appl Acoust 1994;42:29-40.

[2] Muradali A, Fyfe KR. A study of 2D and 3D barrier insertion loss using improved diffraction-based methods. Appl Acoust 1998;53:49-75.

[3] Filippi P, Dumery G. Etude théorique et numérique de la diffraction par un écran mince. Acustica 1969;21:343-59.

[4] Terai T. On calculation of sound fields around three-dimensional objects by integral equation methods. J Sound Vib 1980;69:71-100.

[5] Kawai Y, Terai T. The application of integral equation methods to the calculation of sound attenuation by barriers. Appl Acoust 1990;31: $101-17$.

[6] Morgan PA, Hothersall DC, Chandler-Wilde SN. Influence of shape 
and absorbing surface - a numerical study of railway barriers. J Sound Vib 1998;217(3):405-17.

[7] Lacerda LA, Wrobel LC, Mansur WJ. A dual boundary element formulation for sound propagation around barriers over an infinite plane. J Sound Vib 1997;202:235-347.

[8] Lacerda LA, Wrobel LC, Power H, Mansur WJ. A novel boundary integral formulation for three-dimensional analysis of thin acoustic barriers over an impedance plane. J Acoust Soc Am 1998;104(2): 671-8.

[9] Tadeu A, Godinho L. 3D wave scattering by a fixed cylindrical inclusion submerged in a fluid medium. Engng Anal Bound Elem 1999; 23:745-56. 